\title{
Zeta Potential of Iron Oxide Pigment Powder Using a Rotating Disk
}

\author{
Paulo Henrique Campos Prado Tavares ${ }^{1}$ and Fernando Soares Lameiras ${ }^{2}$ \\ 1. Thematic Network on Materials Engineering (REDEMAT), Federal University of Ouro Preto (UFOP), Ouro Preto 35400-000, \\ Brazil \\ 2. Development Center of Nuclear Technology (CDTN), National Nuclear Energy Commission (CNEN), Belo Horizonte 30161-970, \\ Brazil
}

Received: September 21. 2012 / Accepted: October 26, 2012 / Published: January 25, 2013.

\begin{abstract}
The zeta potential measurement with a rotating disk (RDZP) is a technique originally developed for disk-shaped planar solid samples. In this work, the feasibility of using this technique for pigments powder was studied. The raw material of these pigments came from the desliming step of banded iron formation ore processing. This tailing contains mainly iron oxides $(\approx 76 \mathrm{wt} \%)$ and particles with average size around $4 \mu \mathrm{m}$, which make it a potential pigment. Two different pigments were investigated. The "as produced" mud (brown hue) and that obtained after thermal treatment at $1,000{ }^{\circ} \mathrm{C}$ (red hue). Characterization tests were carried out by X-ray fluorescence (XRF), X-ray diffraction (XRD), thermogravimetric analysis (TG), differential thermal analysis (DTA), and laser granulometry. Color measurements of the pigments were performed using the CIELab system. The RDZP analyses of the pigments agree well with those reported in the literature using the traditional technique of mass transport by electrophoretic motility. The zeta potential measurements showed that the dispersion of the pigments in ammonium hydroxide solution with $\mathrm{Ph}=14$, ensuring absolute zeta potential values above $500 \mathrm{mV}$, is necessary to avoid pigment agglomeration when preparing styrene-acrylic paints.
\end{abstract}

Key words: Zeta potential, rotating disk, banded iron formation, natural pigments, paints.

\section{Introduction}

The traditional technique for zeta potential $(\xi)$ measurements of particles uses the electrophoretic motility concept. A recently developed technique, called zeta potential by rotating disk (RDZP), has been shown to be useful for disk planar surfaces. It is well established that this technique is simpler and less expensive than the conventional one. In their first published paper about RDZP, Sides and Hoggard [1] presented an equation that relates the $\xi$ of the sample disk with the streaming potential $\left(\phi_{s}\right)$ in its vicinity. For this purpose they solved the Laplace's equation for the potential in hydrodynamic conditions observed to the rotating disk.

Corresponding author: Fernando Soares Lameiras, Dr., research fields: ceramic materials and nuclear fuel. E-mail: fsl@cdtn.br.
Hoggard et al. [2] showed that the $\phi_{s}$ can be measured near a rotating disk when it is immersed in a solution. For this it is necessary that the gap between the working electrode and disk is small and known with accuracy. They showed that the setting with the working electrode placed on the axis of the disk at a distance of $1 \mathrm{~mm}$ works well. The measured $\phi_{s}$ was used to provide the $\xi$ of the sample by integration of Laplace's equation within one radius of the disk surface. An important limitation is working with solution concentration up to $10 \mathrm{mM}$.

Despite of the relevancy of these two previous papers and the results agreeing well to the literature, the authors found some discordance between the theoretical predictions and experimental data. This problem was solved in the next paper using the Hankel transform [3].

Despite of the relevance of these two previous 
papers and the results agreeing well to the literature, the authors found some discordance between the theoretical predictions and experimental data. This problem was solved in the next paper using the Hankel transform [3]. Lameiras and Nunes [4] presented an alternative and easier way to calculate the $\phi_{s}$ in the vicinity of a disk-shaped sample rotating in an electrolytic solution. They solved the Chebyshev's equation using rotational elliptic coordinates. They applied the RDZP technique to obtain $\xi$ of some materials of general use [5].

The RDZP technique has been shown very useful to describe the influence of the $\mathrm{pH}$ on $\xi$ for planar surface materials. However, no published study about the possibility to use the RDZP for powder materials was found. In this paper, the RDZP technique was employed to a powder called "mud" by the mining sector. This mud consists of a blend of iron oxides, quartz, and aluminosilicate and it is generated in a desliming stage of the banded iron formations exploitation. This ore containing low content of iron $(\approx$ $45 \mathrm{wt} \%$ ) requires a process of flotation to raise the iron content to $60-65 \mathrm{wt} \%$. For this, a prior desliming step is required to eliminate particles with size below than $10 \mu \mathrm{m}$. Because of the small size distribution and the chemical composition, this tailing had been considered as a raw material for the production of iron oxide pigments. These are one of the most important inorganic pigments, it being surpassed only by the titanium oxide production. The worldwide production of iron oxide pigments is above one million tons per year [6].

Synthetic iron oxides pigments are obtained by the Penniman process that was developed in the 1930's and uses metallic iron as raw material [7]. High quality and uniform iron oxides pigments are produced for the automobile and building industries, as well as for other industrial fields. However, this process uses a series of complex chemical steps that involve the use of acids and bases for oxidation and precipitation reactions.

Pigments obtained from natural minerals are also a source to achieve the market standards. Nonetheless, they are inherently heterogeneous and associated with other minerals. Their broad size distribution and the presence of impurities are critical. The $\xi$ of these pigments is not usually evaluated, although it may provide valuable information about their agglomeration behavior in a dispersant medium. Thus, in this paper we also show the relevance of assessing the $\xi$ to obtain a stable dispersion of pigments in styrene-acrylic aqueous resins.

\section{Experiments}

Two pigments were studied in this work. The mud, "as produced", and after heating in air at $1,000{ }^{\circ} \mathrm{C}$ for 30 minutes. In this work they were called natural and calcined pigments, respectively.

XRF was performed with a dispersive energy spectrometer (Shimadzu EDX-720), under vacuum and a $3 \mathrm{~mm}$ collimator. XRD was performed with a Geigerflex diffractometer (Rigaku), using $\mathrm{Cu} K_{\alpha}$ radiation, $40 \mathrm{kV}, 30 \mathrm{~mA}$, and scanning velocity $(2 \theta)$ of $8 \%$ min.

TG and DTA tests were carried out using DSC-50 and TGA-50 Shimadzu apparatus, respectively. Both tests were performed using a heating rate of $10{ }^{\circ} \mathrm{C} / \mathrm{min}$ and under nitrogen atmosphere $(20 \mathrm{~mL} / \mathrm{min})$. Particle size distribution tests were carried out in a CILAS 1064 granulometer. The samples were dispersed in deionized water in these tests.

The reflectance $L^{*} a^{*} b^{*}$ values of the powder pigments were obtained with a portable spectrometer (StellarNet modelo EPP 2000). The apparatus uses an illumination source D65 (420 nm) that simulates the daylight and also a standard white color (RS 50) as reference. The reflectance probe (R400-7) was connected to a support, located at a distance of $6.0 \mathrm{~mm}$ and 45 degrees from the sample. The measurements were taken using $1.0 \mathrm{~nm}$ resolution, integration time of $100 \mathrm{~ms}$ and 4 scans.

The streaming potential $\left(\phi_{s}\right)$ was measured with a rotating disk(Fig. 1). The face of the cylindrical 


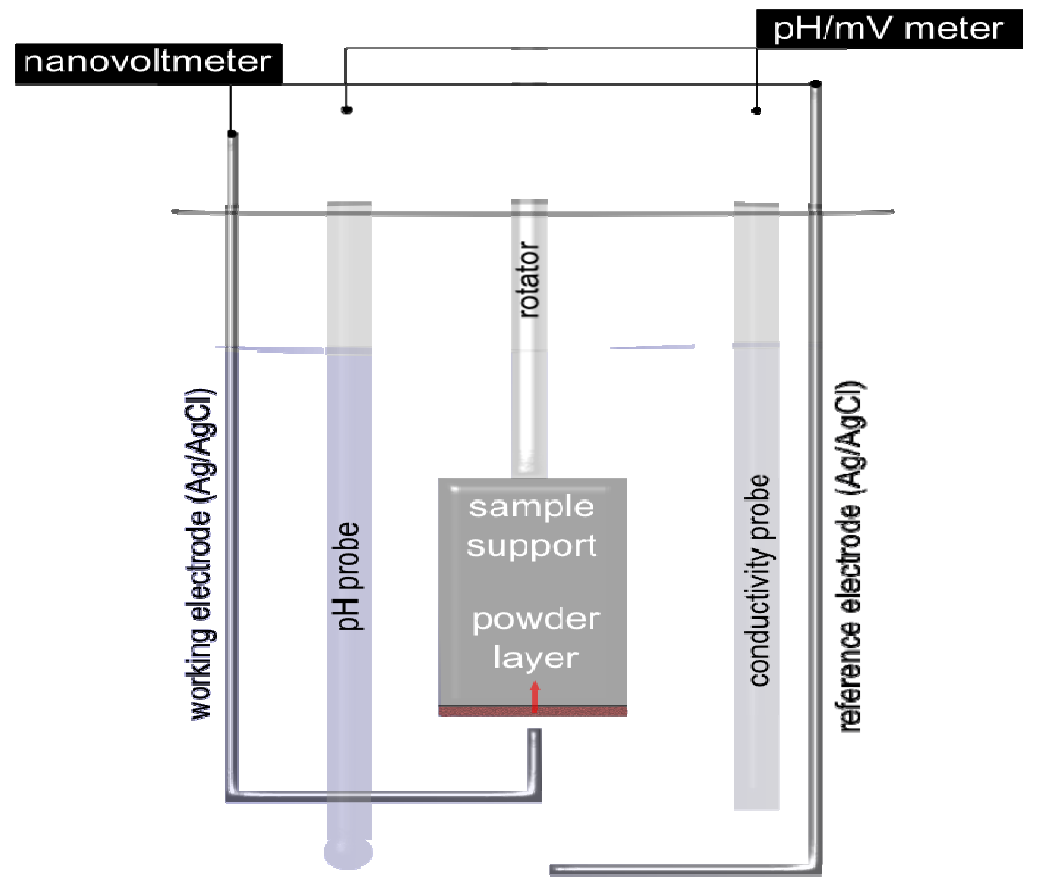

Fig. 1 Schematic of the streaming potential experimental apparatus.

sample support with $1.27 \mathrm{~cm}$ radius $(\alpha)$ was covered with a double sided adhesive tape. The pigment powder was deposited on the tape by manual compression. Afterwards a force of $9.8 \times 10^{3} \mathrm{~N}$ was applied for 5 minutes using a mechanical press. A uniform film of powder was obtained. This set was spun in deionized water at 3,000 rpm to remove the powder excess and the suspension was discharged. The cell was filled with deionized water and this liquid was used as the dispersion medium of the electric charges.

The dielectric constant $(\epsilon)$ was taken as 78.3, the kinematic viscosity $(v)$ as $1 \times 10^{-6} \mathrm{~m}^{2} / \mathrm{s}$, and rotation speed $(\Omega)$ as 3,000 rpm. The working electrode/powder layer gap (z) was $1 \mathrm{~mm}$ and the reference electrode was placed at the bottom of the vessel. The $\mathrm{pH}$ and conductivity of the liquid were taken from the respective probes. The $\mathrm{pH}$ was controlled by adding $0.05 \mathrm{M}$ solutions of $\mathrm{HCl}$ and $\mathrm{KOH}$. The measurements were repeated five times.

The potential measured between the working and reference electrodes is the $\phi_{s}$. The Eq. (1) converts the measured $\phi_{s}$ to zeta potential $(\xi)$.

$$
\xi \cong \frac{1.96 k v^{1 / 2}}{\epsilon a \Omega^{3 / 2}} \frac{1}{2\left(1-\frac{z}{a}-\frac{1}{2\left(\frac{z^{2}}{a^{2}}+1\right)^{1 / 2}}\right)} \phi_{S}
$$

The paints were prepared by adding $1 \mathrm{wt} \%$ of the pigments to a commercial aqueous dispersion of styrene-acrylic resins (Suvinil Acrilic Premium, base C). The pigments were previously dispersed in ammonium hydroxide solutions (Vetec AP) and added to the resin. The homogenization was performed using a dispersing machine with toothed disk at 3,000 rpm.

The visual inspection relative to agglomeration was made after applying three coats of paints on a white paper. Digital images were obtained with a stereomicroscope (2000-C Zeiss). The $\mathrm{pH}$ analysis of the paints were carried out with a $\mathrm{pH}$-meter (Accumet AP85, Fisher Scientific) equipped with a special electrode for dispersions (DME-CV8 Digimed).

\section{Results and Discussion}

The XRF tests revealed that the natural pigment is composed by iron, silicon and aluminum oxides with contents around 76, 16 and $6 \mathrm{wt} \%$, respectively. The 
major phases observed in the XRD pattern of the natural pigment are goethite, hematite, quartz, muscovite, and kaolinite (Fig. 2).

TG tests of the natural pigment revealed a water loss of $0.6 \%$ up to $200{ }^{\circ} \mathrm{C}$ due the moisture. The mass loss of $4.0 \%$ at $322{ }^{\circ} \mathrm{C}$ was associated with an endothermic peak in the DTA curve. This behavior was attributed to a goethite/hematite conversion by dehydroxylation. This reaction usually occurs at $350{ }^{\circ} \mathrm{C}$ in water-free environments and when high heating rates are used. At $522{ }^{\circ} \mathrm{C}$ a mass loss of $1.2 \%$ also occurred due to the endothermic conversion of kaolinite into metakaolin by dehydroxylation [8] (Eqs. (2) and (3)).

$$
2 \mathrm{FeOOH} \rightarrow \mathrm{Fe}_{2} \mathrm{O}_{3}+\mathrm{H}_{2} \mathrm{O} \uparrow
$$

$$
2 \mathrm{SiO}_{2} \cdot \mathrm{Al}_{2} \mathrm{O}_{3} \cdot 2 \mathrm{H}_{2} \mathrm{O} \rightarrow 2 \mathrm{SiO}_{2} \cdot \mathrm{Al}_{2} \mathrm{O}_{3}+2 \mathrm{H}_{2} \mathrm{O} \uparrow
$$

The approximate content of each crystalline phase observed in the natural pigment was obtained by stoichiometric calculations associating the mass losses with the oxide contents from the XRF analysis (Table 1).

The mineralogical composition agrees with that reported by Pires et al. [9]. The total sum above $100 \%$ can be related to the presence of goethite with high content of water (up to $14 \%$ ), substantially more than the $10.1 \%$ expected by the chemical formula of this iron oxide [10]. All crystalline phases found are compatible with paints industry. Besides the iron oxide pigments, the muscovite presents a lamellar particle

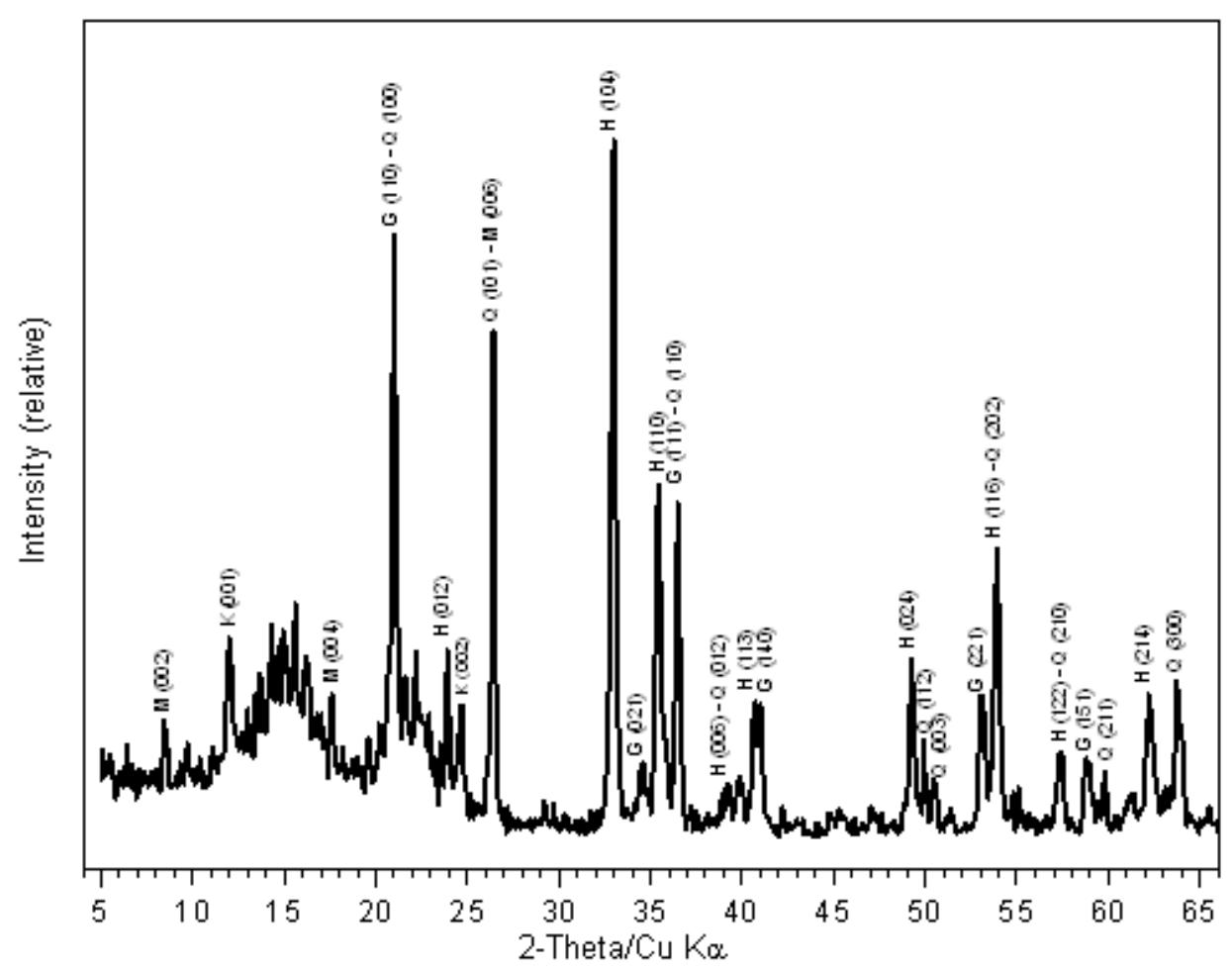

Fig. 2 XRD pattern of the natural pigment. Hematite (H); Goethite (G); Quartz (Q); Kaolinite (K) and Muscovite (M).

Table 1 Semi-quantitative mineralogical composition of the natural pigment.

\begin{tabular}{llc}
\hline Phase & Formula & Content (wt \%) \\
\hline Hematite & $\mathrm{Fe}_{2} \mathrm{O}_{3}$ & 40.4 \\
Goethite & $\mathrm{FeOOH}$ & 39.6 \\
Quartz & $\mathrm{SiO}_{2}$ & 9.2 \\
Muscovite & $\mathrm{KAl}_{2} \mathrm{Si}_{3} \mathrm{AlO}_{10}(\mathrm{OH})_{2}$ & 6.3 \\
Kaolinite & $2 \mathrm{SiO}_{2} \cdot \mathrm{Al}_{2} \mathrm{O}_{3} \cdot 2 \mathrm{H}_{2} \mathrm{O}$ & 8.6 \\
\hline Total & - & 104.1 \\
\hline
\end{tabular}




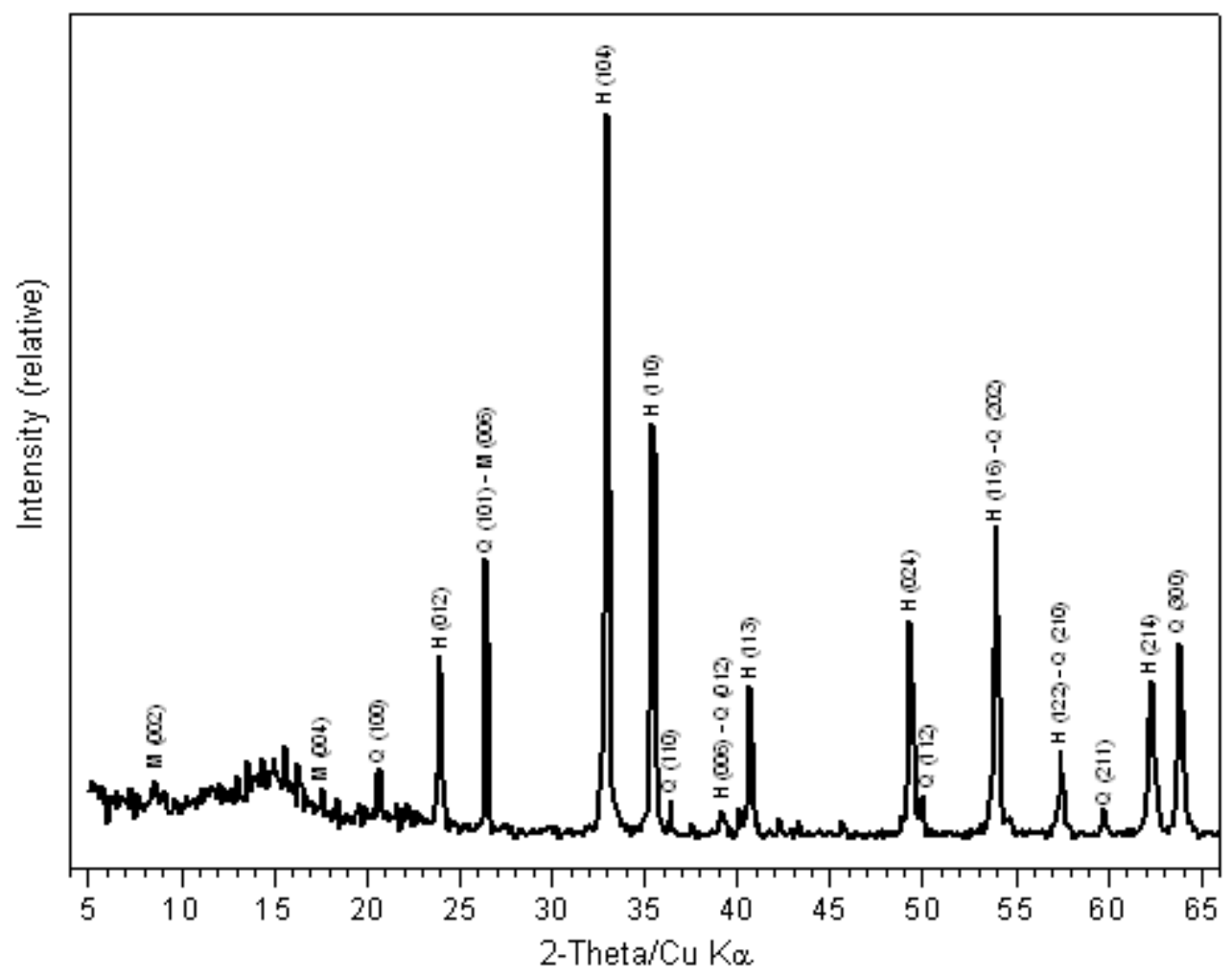

Fig. 3 XRD pattern of the calcined pigment. Hematite (H); Quartz (Q) and Muscovite (M).

shape that affects the paint viscosity, reducing sedimentation [11]. The kaolinite is mainly used to promote opacity and eggshell appearance in paints [12]. The quartz particles enhance the paint brush ability and also its abrasion resistance.

XRD tests of the calcined pigment (Fig. 3) revealed an increase in the intensity of the reflection lines related to hematite, whereas those ascribed to goethite and kaolinite were not observed. These findings are in agreement with the calcination reactions showed previously.

The calcination at $1,000{ }^{\circ} \mathrm{C}$ changed the pigment hue from brown to red due to a goethite/hematite conversion (Fig. 4).

The $L^{*} a^{*} b^{*}$ values of the pigments are given in Table 2. In the CIELab color space the positive $a^{*}$ axis points in the direction of red color stimulus; negative $a^{*}$ (green); positive $b^{*}$ (yellow) and negative $b^{*}$ (blue). $L^{*}$ is associated with the lightness [13]. Natural pigment has a brown hue due the mixing of the red (from hematite) and yellow (from goethite) stimuli. For calcined pigment, besides the increase in the red stimulus, the yellow one dropped practically to zero. This explains the red hue observed to the calcined pigment in Fig. 4 and totally agrees with the diffraction analyses. The change in $L^{*}$ was not significant.

The natural and calcined pigments presented a medium grain size of 4.0 and $5.0 \mu \mathrm{m}$, respectively. There was no retention in the wet sieving using a mesh with square hole of $38 \mu \mathrm{m}$. Pigments with grain size in the order of tens micrometers usually have good
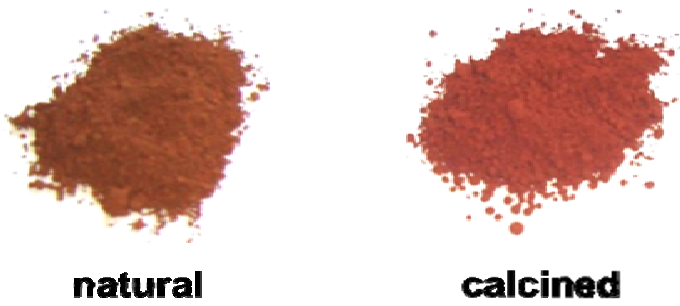

Fig. 4 Photograph of pigments with different colors.

Table $2 L^{*} a * b *$ chromatic parameters.

\begin{tabular}{llll}
\hline Pigment & $L^{*}$ & $a^{*}$ & $b^{*}$ \\
\hline Natural & 82.26 & 7.04 & 1.39 \\
Calcined & 80.06 & 7.50 & 0.11 \\
\hline
\end{tabular}


properties like high tinting strength and hiding [14]. This means that the pigments size distribution is suitable for painting industry.

The pictures of the paints coatings are shown in Fig. 5. The pigments were pre-dispersed in ammonium hydroxide solution of $\mathrm{pH}=8.0$. There was agglomeration of the pigment particles in both cases. Some agglomerates were $500 \mu \mathrm{m}$ in size and the finishing do not match with the industrial standards. The $\mathrm{pH}$ of the paints prepared in this way was the same of the hydroxyl solution.

Zeta potential $(\xi)$ measurements of the pigments powder were performed in order to investigate the agglomeration problem (Fig. 6). There was a pronounced decrease in $\xi$ values for pH's above 12. This result agrees reasonably well with that described by Lima and Quirino [15] for hematite using a mass transport camera. Moreover, the isoelectric point is not between $\mathrm{pH}=6-10$, as expected for iron oxides. This

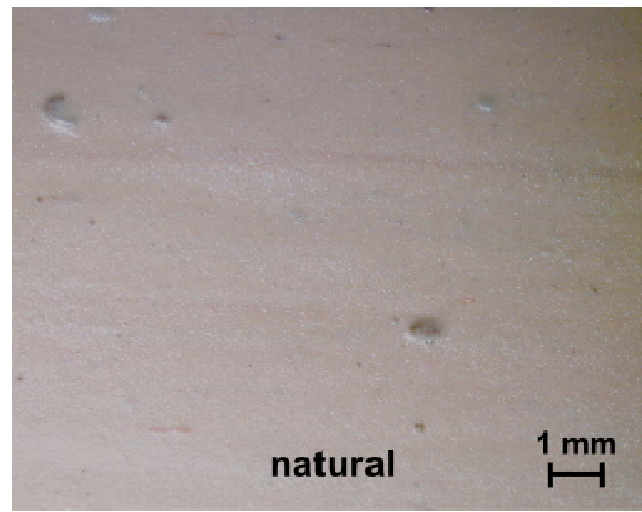

was attributed to the silica content in the samples which has the isoelectric point for $\mathrm{pH}<3$ [16]. The poor reproductions observed in $\xi$ measurements for $\mathrm{pH}$ 's above 12 can be associated with the large increase in the solution conductivity due the $\mathrm{KOH}$ additions. The higher the conductivity, the lower the streaming potential signals. This behavior leads to the decrease of the analysis sensibility.

In order to avoid agglomeration, pigments powder was pre-dispersed in ammonium hydroxide solution with $\mathrm{pH}=14$. This solution is usually employed to alkalinize water-based paints. Under these conditions, the RDZP analysis ensures $\xi<-500 \mathrm{mV}$. There should be sufficient negative charges on the particles surface to keep them apart from each other. The $\mathrm{pH}$ of the paints prepared was 10.3. The pictures of the alkaline paints coatings are shown in Fig. 7. As expected by the RDZP results, there was no significant agglomeration observed in the paints coats.

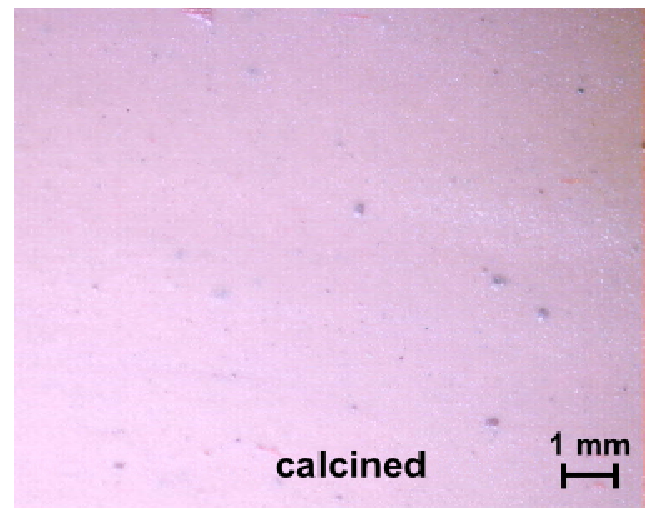

Fig. 5 Photographs of the paints coatings $(\mathrm{pH}=8.0)$ showing the agglomeration problem.

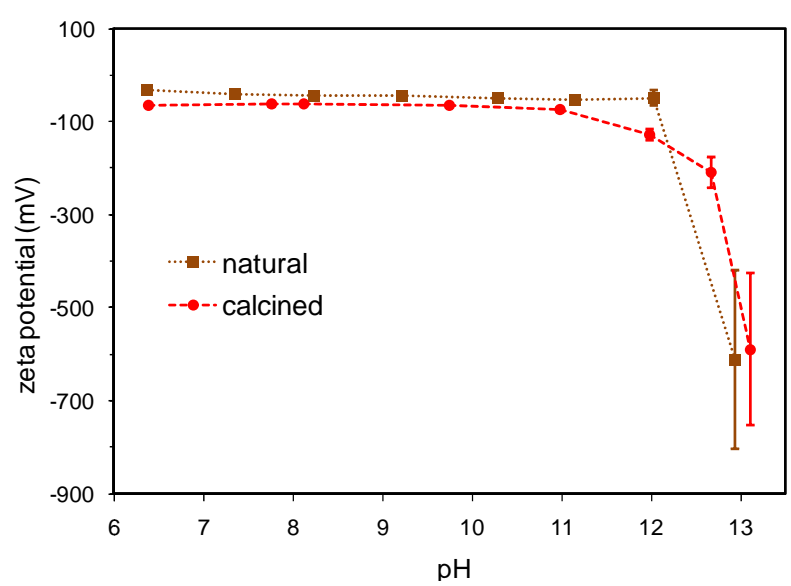

Fig. 6 Zeta potential of pigments used in this work as a function of solution $\mathrm{pH}$. 


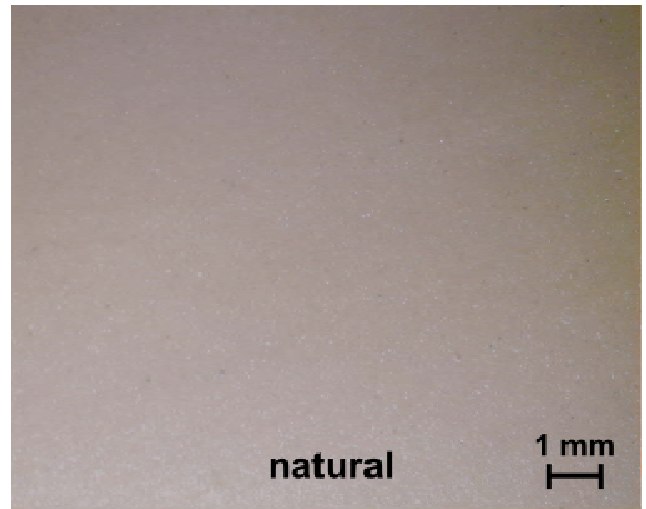

Fig. 7 Photographs of the paints coatings $(\mathrm{pH}=10.3)$.

\section{Conclusions}

The powder method was successfully used to study the zeta potential $(\xi)$ of iron oxide pigments by means of RDZP technique. Pigment stable suspensions in styrene-acrylic resins were obtained after control the $\xi$ of the particles with ammonium hydroxide solution. Thus the powder method applied to RDZP is feasible and useful for the quality control on paints industry.

\section{Acknowledgments}

The authors are grateful to CNPq and FAPEMIG for the financial support; CDTN for the laboratorial facilities and Samarco Mineração S. A. for the samples and overall aid.

\section{References}

[1] P.J. Sides, J.D. Hoggard, Measurement of the zeta potential of planar solid surfaces by means of a rotating disk, Langmuir 20 (2004) 11493-11498.

[2] J.D. Hoggard, P.J. Sides, D.C. Prieve, Measurement of the streaming potential and streaming current near a rotating disk to determine its zeta potential, Langmuir 21 (2005) 7433-7438.

[3] P.J. Sides, J. Newman, J.D. Hoggard, D.C. Prieve, Calculation of the streaming potential near a rotating disk, Langmuir 22 (2006) 9765-9769.

[4] F.S. Lameiras, E.H.M. Nunes, Calculation of the streaming potential near a rotating disk with rotational elliptic coordinates, Portugaliae Electrochimica Acta 26 (4) (2008) 369-375.

[5] F.S. Lameiras, A.L. de Souza, V.A.R. de Melo, E.H.M. Nunes, I.D. Braga, Measurement of the zeta potential of planar surfaces with a rotating disk, Materials Research 11 (2) (2008) 217-219.

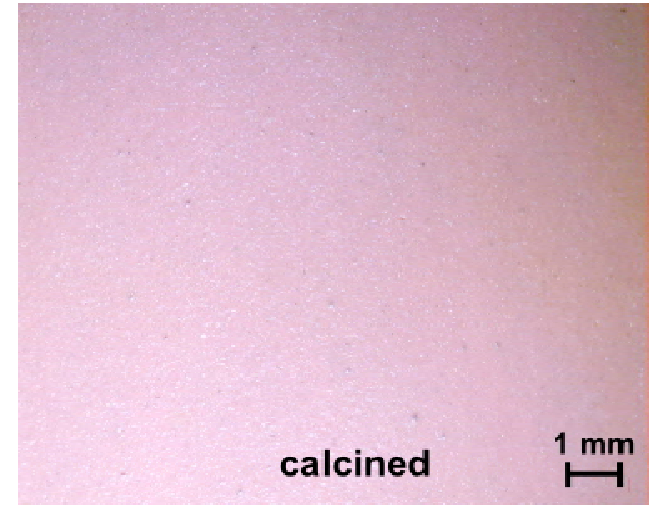

[6] N. Guskos, G.J. Papadopoulosa, V. Likodimosa, S. Patapisa, D. Yarmisa, A. Przepierab, et al., Photoacoustic, EPR and electrical conductivity investigations of three synthetic mineral pigments: Hematite, goethite and magnetite, Materials Research Bulletin 37 (2002) 1051-1061.

[7] R.M. Cornell, U. Schwertmann, The Iron Oxides: Structure, Properties, Reactions, Occurrence and Uses, (2nd Ed.) Wiley-VCH, Weinheim, 1996, pp. 525-540.

[8] B.S. Carneiro, R.S. Angélica, T. Scheller, E.A.S. de Castro, R.F. Neves, Mineralogical and geochemical characterization of the hard kaolin from the Capim region, Pará, northern Brazil, Cerâmica 49 (2003) 237-244.

[9] J.M.M. Pires, J.C. Lena, C.C. Machado, R.S. Pereira, Polluting potential of samarco mineração s.a. solid waste: A germano dam case study, R. Árvore 27 (2003) 393-397.

[10] S.G. Elizalde, M.E.G. Clavel, Thermal-behavior in air of iron oxyhydroxides obtained from the method of homogeneous precipitation: Part 1. Goethite samples of varying crystallinity, Thermochimica Acta 124 (1988) 359-369.

[11] Kalendová, D. Veselý, P. Kalenda, Properties of paints with hematite coated muscovite and talc particles, Applied Clay Science 48 (2010) 581-588.

[12] A.A. Tracton, Coatings Technology Handbook, (3rd Ed.), CRC Press, Boca Raton, 2006, pp. 82-83.

[13] J. Schanda, Colorimetry, Understanding the CIE System, Wiley, Hoboken, 2007, p. 62.

[14] M.A. Legodi, D. Waal, The preparation of magnetite, goethite, hematite and maghemite of pigment quality from mill scale iron waste, Dyes and Pigments 74 (1) (2007) 161-168.

[15] R.M.F. Lima, L. Quirino, Effect of amine adsorption on zeta potential of hematite and quartz, Escola de Minas Magazine 56 (1) (2003) 45-49.

[16] L. Bousse, S. Mostarshed, B.V.D. Shoot, N.F de Rooij, P. Gimmel, W. Göpel, Zeta potential measurements of $\mathrm{Ta}_{2} \mathrm{O}_{5}$ and $\mathrm{SiO}_{2}$ thin films, Journal of Colloid and Interface Science 147 (1) (1991) 22-32. 